\title{
Translational Regular Variation Asymptotic Behavior and Applications
}

\author{
Milan R. TAsković
}

\begin{abstract}
In this paper we introduce some new classes of functions which are a translational regular asymptotic behavior. In this sense we continue the study of the translational regularly varying functions. This results are closely connected with the Karamata's theory of regularly varying functions.

On the other hand, in this paper we give some theorems of Tauberian nature via the translational regularly varying functions. Applications of new Tauberian theorems and a method of the Monotone Density theorem for Stieltjes transform are considered.

This results are connection with the Karamata's Tauberian theorems, with the Karamata's Hauptsatz, as and with the classical statements of Hardy and Littlewood.
\end{abstract}

1. Introduction and history. We shall say that a positive, finite and measurable function $K$, defined on $I_{a}:=[a, \infty)$ for some $a>0$, is a regularly varying function at infinity (denoted this class by $R V$ ) in the sense of Karamata if the limit

$$
\lim _{x \rightarrow \infty} \frac{K(\lambda x)}{K(x)}=r(\lambda)
$$

is positive and finite for each $\lambda>0$. It follows immediately that $r(\lambda)=\lambda^{\rho}$ for some $\rho \in \mathbb{R}$. The number $\rho$ is the index of $K$.

The $R V$ functions of index $\rho=0$ are called slowly varying (denoted this class by $S V$ ) functions and are denoted by $L$. Their interest lies in the fact that $K$ is a $R V$ function of index $\rho$ if and only if $K(x)=x^{\rho} L(x)$ for $L \in S V$ on some $I_{a}$. Classes $S V$ and $R V$ of slowly and regularly varying functions were introduced by Jovan Karamata in 1930. In this respect we refer to the

1991 Mathematics Subject Classification. Primary: 26A12, 40E05, 46F10. Secondary: 42A20, 43A85.

Key words and phrases. Translational slowly varying function, Translational regularly varying function, Translational $\mathcal{O}$-regularly varying function, uniform convergence, characterization, representation, Slowly varying function, Regularly varying function, $\mathcal{O}$-regularly varying function, Karamata's theory, Karamata's Hauptsatz, Tauberian theorems, Theorems of Tauberian nature, Stieltjes transform, Laplace-Stieltjes transform, Monotone Density Theorem, Karamata's Tauberian theorems, Hardy- Littlewood theorems. 
books of E. Seneta [28] and Bingham-Goldie-Teugels [4]. Both classes have important consequences in the study of asymptotic processes.

A positive, finite and measurable function $f$, defined on $I_{a}$ for some $a>0$, is said to be translational regularly varying at infinity (denoted this class by $\operatorname{Tr}(R V))$ if the limit

$$
\lim _{x \rightarrow \infty} \frac{f(x+\lambda)}{f(x)}=h(\lambda)
$$

is positive and finite for each $\lambda \geq 0$. In actual fact a weaker definition can be used, for the assumption that this positive finite limit property obtains for all $\lambda$ in a subset of positive measure of $(0, \infty)$ implies that it obtains for all $\lambda \in(0, \infty)$.

In connection with this, since $h(\lambda)$ is a positive measurable solution of the following functional equation

$$
h(u+v)=h(u) h(v), \quad u, v>0,
$$

it is well known that $h(\lambda)=e^{\sigma \lambda}$ for some finite $\sigma$, and so we can write $f(x)=e^{\sigma \lambda} A(x)$, where

$$
\lim _{x \rightarrow \infty} \frac{A(x+\lambda)}{A(x)}=1 \text { for each } \lambda \geq 0 ;
$$

such a translational regularly varying function, for which the index $\sigma$ of translational regular variation is zero, is called translational slowly varying (denoted this class by $\operatorname{Tr}(S V)$ ). Classes $\operatorname{Tr}(S V)$ and $\operatorname{Tr}(R V)$ of translational slowly and regularly varying functions were introduced by M. Tasković in [32]. The three most important properties of translational regularly varying functions (from which others are easily deducible) are:

(i) The convergence in (1), or equivalently (3), is uniform for $\lambda$ in any fixed interval $[a, b], 0<a<b<\infty$.

(ii) (Representation Theorem). There exist $\sigma \in \mathbb{R}$ and a number $b \geq a$ such that for $x \geq b$ a translational regularly varying function $f$ has representation

$$
f(x)=\mu(x) \exp \left(\sigma x+\int_{b}^{x} \varepsilon(t) d t\right),
$$

where $\mu(x)$ is a positive and measurable function on $I_{b}$ such that $\mu(x) \rightarrow$ $c \in(0, \infty)$ as $x \rightarrow \infty$, and $\varepsilon(x)$ is a continuous function on $I_{b}$ such that $\varepsilon(x) \rightarrow 0($ as $x \rightarrow \infty)$.

Conversely, any function $f$ having representation (4) is clearly translational regularly varying.

(iii) (Characterization Theorem). If $f$ is a translational regularly varying function, then the limit $h(\lambda)$ in (1) is necessarily of the form $e^{\sigma \lambda}$ for some $-\infty<\sigma<\infty$ and for each $\lambda \geq 0$.

In connection with the Karamata's functions we notice that Bojanić and Karamata in 1963 are have been considered for a real valued function $f$ the 
following equality

$$
\lim _{x \rightarrow \infty} \frac{f(\lambda x)-f(x)}{g(x)}=H(\lambda),
$$

for every $\lambda>1$, where $g \in R V$, i.e., $g(x)=x^{\sigma} L(x)$ for some finite $\sigma$ and $L \in S V$. This class of all functions $f$ is denoted by $K B$.

The last condition played an important role in papers: L. de Haan [10]; Ash, Erdös and Rubel [1]; Bingham and Goldie [2,3]; Omey [24]; Delange [9]; Geluk, L. de Haan, and Stadtmüller [31]; and in many others.

In this paper we continue the study of the translational regularly varying functions via a translational regularly varying behaviour. In this sense we introduce and considered some new classes of functions with the preceding translational behaviour.

2. Translational regularly varying behaviour. Let $x \mapsto G(x)$ be a measurable positive function on $I_{a}$ for some $a>0$, and $f$ be a real valued function, defined for sufficiently large positive value of its argument such that the limit

$$
\lim _{x \rightarrow \infty} \frac{f(x+\lambda)-f(x)}{G(x)}=R(\lambda)
$$

exists for all $\lambda$ in a subset $S_{1} \subset[0, \infty)$ of positive measure, and that there is a subset $S_{2}$ (still of positive measure) of $S_{1}$, and a number $\lambda_{1} \in S_{1}$ such that $R\left(\lambda_{1}\right) \neq 0$ with $\mu \in S_{2}$ implies $\lambda_{1}+\mu \in S_{1}$ and $R\left(\lambda_{1}+\mu\right)-R(\mu) \neq 0$.

The class of all functions $f$ which satisfying the preceding condition $(\mathrm{Tb})$ is denoted by $\operatorname{Tr}(K B)$. The following fact is essential.

Proposition 1. If $f \in \operatorname{Tr}(K B)$, then $x \mapsto G(x)$ is a translational regularly varying function, i.e., $G(x)=e^{\sigma x} A(x)$ for some $-\infty<\sigma<\infty$ and $A \in \operatorname{Tr}(S V)$.

Proof. Let $f \in \operatorname{Tr}(K B)$, then for $\mu \in S_{2}$ we obtain the following essential equality of the form

$$
\frac{f(x+\lambda+\mu)-f(x)}{G(x)}=\frac{f(x+\lambda+\mu)-f(x+\mu)}{G(x+\mu)} \cdot \frac{G(x+\mu)}{G(x)}+\frac{f(x+\mu)-f(x)}{G(x)},
$$

hence, as $x \mapsto \infty$, we obtain

$$
R(\lambda+\mu)=R(\lambda) \lim _{x \rightarrow \infty} \frac{G(x+\mu)}{G(x)}+R(\mu),
$$

where this limit must exist and in fact that

$$
\lim _{x \rightarrow \infty} \frac{G(x+\mu)}{G(x)}=\frac{R(\lambda+\mu)-R(\mu)}{R(\lambda)}
$$

exists for every $\mu \in S_{2}$, and being nonzero here, is strictly positive. Hence, by Characterization Theorem for the clas $\operatorname{Tr}(R V)$ (see: Tasković [32]), $G$ is 
a function of translational regular variation, i.e., $G(x)=e^{\sigma x} A(x)$ for some finite $\sigma$ and $A \in \operatorname{Tr}(S V)$. The proof is complete.

In connection with this statement, in further, the class of all functions $f$ which satisfying the condition (Tb) for $G(x)=e^{\sigma x} A(x)$ is denoted by $\operatorname{Tr}\left(K B_{\sigma}\right)$ for $-\infty<\sigma<\infty$.

Theorem 1. (Characterization Theorem). If $f \in \operatorname{Tr}(K B)$, then the limit $R(\lambda)$ in (Tb) is necessarily of the form

$$
R(\lambda)=C\left(1-e^{\sigma \lambda}\right)
$$

for $\sigma \neq 0$ and some constant $C \neq 0$. If $f \in \operatorname{Tr}(K B)$ is a measurable function on $I_{a}$ for some $a>0$ and $\sigma=0$, then

$$
R(\lambda)=c \lambda \quad(\text { for some constant } c \neq 0) .
$$

Proof. As well known, since $S_{1}$ is a set of positive measure, from a result of Steinhaus [30], we can find a number $b>1$ such that for any $\lambda \in[1, b]$ there exist $\alpha, \beta \in S_{1}$ such that $\lambda=\beta-\alpha$. Thus for any $\mu \in[1, b]$ there is an $\lambda_{2}$ such that $\mu+\lambda_{2} \in S_{1}$. Hence for $\lambda \in[1, b]$ we have

$$
\frac{f(x+\lambda)-f(x)}{G(x)}=\frac{f\left(\lambda+\lambda_{2}+x-\lambda_{2}\right)-f\left(\lambda_{2}+x-\lambda_{2}\right)}{e^{\sigma \lambda_{2}} e^{\sigma\left(x-\lambda_{2}\right)} A\left(\lambda_{2}+x-\lambda_{2}\right)}
$$

and putting $y=x-\lambda_{2}$ we obtain

$$
\frac{f(x+\lambda)-f(x)}{G(x)}=\frac{f\left(y+\lambda+\lambda_{2}\right)-f(y)}{e^{\sigma \lambda_{2}} e^{\sigma y} A\left(y+\lambda_{2}\right)}-\frac{f\left(y+\lambda_{2}\right)-f(y)}{e^{\sigma \lambda_{2}} e^{\sigma y} A\left(y+\lambda_{2}\right)},
$$

whence as $y \rightarrow \infty$, since $A\left(y+\lambda_{2}\right) \sim A(y)$ and $\lambda+\lambda_{2} \in S_{1}$, we obtain

$$
\lim _{x \rightarrow \infty} \frac{f(x+\lambda)-f(x)}{G(x)}=e^{-\sigma \lambda_{2}}\left(R\left(\lambda+\lambda_{2}\right)-R\left(\lambda_{2}\right)\right)
$$

as a finite number for arbitrary $\lambda \in[1, b]$. It is now necessary to extend this to $\lambda \in[0, \infty))$.

In this sense let $\gamma \geq 0$ and take fixed $\lambda \in[1, b]$. Then, similarly with the preceding, we have

$$
\begin{gathered}
\frac{f(x+\gamma)-f(x)}{G(x)}=\frac{f(\lambda+\gamma+x-\lambda)-f(\gamma+x-\lambda)}{e^{\sigma(\gamma+x-\lambda)} e^{\sigma(\lambda-\gamma)} A(\lambda-\gamma+\gamma+x-\lambda)}- \\
-\frac{f(\lambda-\gamma+\gamma+x-\lambda)-f(\gamma+x-\lambda)}{e^{\sigma(\gamma+x-\lambda)} e^{\sigma(\lambda-\gamma)} A(\lambda-\gamma+\gamma+x-\lambda)}
\end{gathered}
$$

and putting $y=\gamma+x-\lambda$

$$
=\frac{f(y+\lambda)-f(y)}{e^{\sigma(\lambda-\gamma)} e^{\sigma y} A(y+\lambda-\gamma)}-\frac{f(y+\lambda-\gamma)-f(y)}{e^{\sigma(\lambda-\gamma)} e^{\sigma y} A(y+\lambda-\gamma)},
$$

thus, if $\lambda-\gamma \in[1, b]$, letting $y \rightarrow \infty$ we obtain

$$
\lim _{x \rightarrow \infty} \frac{f(x+\gamma)-f(x)}{G(x)}=e^{-\sigma(\lambda-\gamma)}(R(\lambda)-R(\lambda-\gamma)),
$$


i.e., this means that for $0 \leq \gamma<\lambda-1$, i.e., for all $\gamma$ satisfying $0 \leq \gamma<b$, that

$$
\lim _{x \rightarrow \infty} \frac{f(x+\gamma)-f(x)}{G(x)}=R(\gamma)
$$

exists and is finite. Repeating the argument $(k-1)$ times, say, we obtain that (6) obtains for all $\gamma$ satisfying $0 \leq \gamma<b^{k}$. Since $b>1$, we have that any positive $\gamma$ will eventually be convered by this interval.

Assuming $\sigma \neq 0$, proceed as in the preceding proof of Proposition 1, except using arbitrary $\lambda$ in place of $\lambda_{1}$ and arbitrary $\mu$, to get

$$
R(\lambda+\mu)=e^{\sigma \lambda} R(\mu)+R(\lambda),
$$

so that, interchanging the role of $\lambda$ and $\mu$, on the other hand, we obtain

$$
R(\lambda+\mu)=e^{\sigma \mu} R(\lambda)+R(\mu)
$$

whence, equating (7) and (8) we have the following fact that is

$$
R(\lambda)\left(1-e^{\sigma \mu}\right)=R(\mu)\left(1-e^{\sigma \lambda}\right),
$$

i.e., supposing $\lambda, \mu \neq 0$ we obtain

$$
R(\lambda)=C\left(1-e^{\sigma \lambda}\right),
$$

where $C=$ constant $=R(\mu) /\left(1-e^{\sigma \mu}\right)$. The equality (9) is correct and for $\lambda=0$ also.

In the second case, if $\sigma=0$ and $f$ is assumed measurable on $I_{a}$ for some $a>0$, then $R(\lambda)$ is a measurable function on $(0, \infty)$ such that

$$
R(\lambda+\mu)=R(\lambda)+R(\mu)
$$

for $\lambda, \mu>0$. Thus $R(\lambda)=c \lambda(c \neq 0)$ is a finite, measurable and positive solution for $\lambda>0$ to the Cauchy functional equation (10). The proof is complete.

In connection with the preceding Proposition 1 and Theorem 1, we notice that, if we are willing to assume nature of $x \mapsto G(x)$ in $(\mathrm{Tb})$, then, in place of Theorem 1, we may consider the following neater statement, which requires no further proof. This is similar stroke like in: Seneta [28].

Theorem 2. Let $f$ be a real valued function, defined on $I_{a}$ for some $a>0$, and $A \in \operatorname{Tr}(S V)$ such that

$$
\lim _{x \rightarrow \infty} \frac{f(x+\lambda)-f(x)}{e^{\sigma x} A(x)}=R(\lambda)
$$

exists (is finite) for $\lambda$ in a subset $S \subset[0, \infty)$ of positive measure. Then (11) obtains with a finite limit $R(\lambda)$ for every $\lambda \in[0, \infty)$. If $\sigma \neq 0$, then

$$
R(\lambda)=C\left(1-e^{\sigma \lambda}\right)
$$

for some constant $C$. If $\sigma=0$ and $f$ is assumed measurable on $I_{a}$, then

$$
R(\lambda)=c \lambda \quad(\text { for some constant } c) .
$$


Suppose additionally that, in the case $\sigma>0 f$ is bounded on each finite interval beyond a certain point, then if $\sigma>0$ :

$$
f(x)=C e^{\sigma x} A(x)+o\left(e^{\sigma x} A(x)\right), \quad \text { as } x \mapsto \infty,
$$

and if $\sigma<0$;

$$
f(x)=K+C e^{\sigma x} A(x)+o\left(e^{\sigma x} A(x)\right), \quad \text { as } \quad x \rightarrow \infty,
$$

where the constant $K=\lim _{x \rightarrow \infty} f(x)$ exists.

The most important property of $\operatorname{Tr}(K B)$ functions may be stated as follows as the analog of uniformity of convergence on finite intervals.

Theorem 3. (Uniform Convergence Theorem). Let $f$ be a real valued measurable function, defined for sufficiently large values on $I_{a}$ for some $a>0$, and $G(x)=e^{\sigma x} A(x)$ for $-\infty<\sigma<\infty$ and $A \in \operatorname{Tr}(S V)$ such that

$$
f(x+\lambda)-f(x)=O(G(x)), \quad \text { as } x \rightarrow \infty,
$$

for each $\lambda \geq 0$, then this property holds uniformly with respect to $\lambda \in[\alpha, \beta]$ for $0 \leq \alpha<\beta<\infty$.

The statement remains true if (12) is replaced by

$$
f(x+\lambda)-f(x)=o(G(x)), \quad \text { as } x \rightarrow \infty .
$$

Proof. We prove uniformity with respect to $\mu \in[0,1]$. If this does not hold then there exist sequences $\left\{x_{n}\right\}_{n \in \mathbb{N}}$ with the property $x_{n} \rightarrow \infty$ and $\left\{\mu_{n}\right\}_{n \in \mathbb{N}}$ in $[0,1]$ such that

$$
\frac{\left|f\left(x_{n}+\mu_{n}\right)-f\left(x_{n}\right)\right|}{G\left(x_{n}\right)} \rightarrow \infty \quad(n \rightarrow \infty)
$$

From Seneta $\left[28\right.$, p.80] there exist measurable sets $U_{r, s}$ and $V_{r, s}$ defined by

$$
U_{r, s}=\left\{\mu \in[0,2]:\left|f\left(x_{n}+\mu\right)-f\left(x_{n}\right)\right|<r G\left(x_{n}\right) \quad \text { for } n \geq s\right\}
$$

and

$V_{r, s}=\left\{\lambda \in[0,2]:\left|f\left(x_{n}+\mu_{n}+\lambda\right)-f\left(x_{n}+\mu_{n}\right)\right|<r G\left(x_{n}+\mu_{n}\right)\right.$ for $\left.n \geq s\right\}$,

where $U_{r, s}$ and $V_{r, s}$ are clearly measurable, each is a monotone increasing sequence of sets as $r \rightarrow \infty$ or $s \rightarrow \infty$; also, $U_{r, s}$ and $V_{r, s}$ converges to [0,2] as $r, s \rightarrow \infty$.

Select $r$ and $s$ large enough that $m\left(U_{r, s}\right)>3 / 2$ and $m\left(V_{r, s}\right)>3 / 2$ with $U_{r, s} \subset[0,2] \subset[0,3]$ and $D_{r, s}=V_{r, s}+\mu_{s} \subset[0,3]$, where $m\left(D_{r, s}\right)>3 / 2$. Thus there is $\mu \in U_{r, s}$ such that $\mu \in V_{r, s}+\mu_{s}$ or $\mu-\mu_{s} \in V_{r, s}$. For this $\mu$ from (14) and (15) we have

$$
\left|f\left(x_{s}+\mu\right)-f\left(x_{s}\right)\right|+\left|f\left(x_{s}+\mu\right)-f\left(x_{s}+\mu_{s}\right)\right|<r\left(G\left(x_{s}\right)+G\left(x_{s}+\mu_{s}\right)\right),
$$


so that, by the triangle inequality, hence we obtain the following inequality

$$
\frac{\left|f\left(x_{s}+\mu_{s}\right)-f\left(x_{s}\right)\right|}{G\left(x_{s}\right)}<r\left(1+\frac{G\left(x_{s}+\mu_{s}\right)}{G\left(x_{s}\right)}\right),
$$

i.e., as $s \rightarrow \infty$, the Uniform Convergence Theorem for translational regularly varying functions gives

$$
\limsup _{s \rightarrow \infty} \frac{\left|f\left(x_{s}+\mu_{s}\right)-f\left(x_{s}\right)\right|}{G\left(x_{s}\right)} \leq r\left(1+e^{\sigma}\right),
$$

since $\mu_{n} \in[0,1], G(x)=e^{\sigma x} A(x)$ and $A \in \operatorname{Tr}(S V)$. But this contradicts to (13). Second part of statement for case $f(x+\lambda)-f(x)=o(G(x))$ can be proved analogously. The proof is complete.

Proposition 2. let $A \in \operatorname{Tr}(S V)$ and let $x \mapsto h(x)$ be an e.g. measurable function on $I_{a}$ for some $a>0$ and bounded on each finite interval sufficiently far, then

$$
A(x) h(x)=A(x) \tau(x)+\int_{a}^{x} \tau(t) A(t) d t,
$$

for $x \geq a$, where

$$
\tau(x)=\frac{1}{x}\left(h(x)-\frac{1}{x A(x)} \int_{a}^{x} h(y) A(y) d y\right) .
$$

Proof. The equality (16) is a consequence of calculation from the following integral equality

$$
\int_{a}^{x}\left(h(t) A(t)-\frac{1}{t} \int_{a}^{t} h(y) A(y) d y\right) \frac{1}{t} d t=\frac{1}{x} \int_{a}^{x} h(y) A(y) d y .
$$

Proposition 3. Let $A$ be a translational slowly varying function, i.e., $A \in$ $\operatorname{Tr}(S V)$, then

$$
\lim _{x \rightarrow \infty} \frac{\int_{a}^{x} A(t) d t}{A(x)}=1 .
$$

Proof. If $b \geq a$ is chosen so that $A$ is bounded on $[b, x]$ for each fixed $x \geq b$, then for $0<\delta<1$ we obtain the following inequalities

$$
\int_{b}^{x} A(t) d t=\int_{b}^{x} e^{-\delta t} e^{\delta t} A(t) d t \leq \sup _{b \leq t \leq x}\left(e^{-\delta t} A(t)\right) \int_{b}^{x} e^{\delta t} d t,
$$

i.e.,

$$
\int_{b}^{x} A(t) d t \leq \sup _{b \leq t \leq x}\left(e^{-\delta t} A(t)\right) \frac{e^{\delta x}-e^{\delta b}}{\delta}
$$

and similarly

$$
\int_{b}^{x} A(t) d t \geq \inf _{b \leq t \leq x}\left(e^{-\delta t} A(t)\right) \frac{e^{\delta x}-e^{\delta b}}{\delta},
$$


Thus, as $x \rightarrow \infty$, the right hand sides of (20) and (21) from the known facts on properties for $\operatorname{Tr}(R V)$ are asymptotic equality with $A(x) / \delta$ and $\delta A(x)$ respectively, i.e., thus we have

$$
\delta \leq \liminf _{x \rightarrow \infty} \frac{\int_{b}^{x} A(t) d t}{A(x)} \leq \limsup _{x \rightarrow \infty} \frac{\int_{b}^{x} A(t) d t}{A(x)} \leq \frac{1}{\delta},
$$

and since $0<\delta<1$ is arbitrary, it follows that statement holds as $\delta \rightarrow 1$. The proof is complete.

Theorem 4. Let $f \in \operatorname{Tr}\left(K B_{0}\right)$ defined on $I_{a}$ for some $a>0$ and $A \in$ $\operatorname{Tr}(S V)$, then for $x \geq a$ the following representation holds

$$
f(x)=\int_{a}^{x} \delta(t) A(t) d t+o(A(x))
$$

as $x \rightarrow \infty$, where $\lim _{x \rightarrow \infty} \delta(x)$ exists and where $R(\lambda)=c \lambda$ for some constant $c$.

Proof. First let $h(x)=f(x) / A(x)$ which is measurable on $I_{a}$ and bounded on finite intervals of $I_{a}$. Put correspondingly $\gamma(x)$ for $\tau(x)$ in (16), then

$$
f(x)=x A(x) \gamma(x)+\int_{a}^{x} \gamma(t) A(t) d t
$$

and a second application of Proposition 2 with $h(x)=x \gamma(x)$ supposing for the moment the requisite boundedness on finite intervals sufficiently far of $\gamma(x)$ and correspondingly $\tau(x)=\varepsilon(x)$ gives

$$
x A(x) \gamma(x)=A(x) \varepsilon(x)+\int_{a}^{x} \varepsilon(t) A(t) d t
$$

such that so combining (22) and (23) we obtain the following fact

$$
f(x)=\int_{a}^{x} \delta(t) A(t) d t+\varepsilon(x) A(x),
$$

where $\delta(x)=\gamma(x)+\varepsilon(x)$. It now suffices to prove $\gamma(x) \rightarrow R(\lambda) / \lambda$ as $x \rightarrow \infty$, for this implies also boundedness of $\gamma$ and $\varepsilon(x) \rightarrow 0$ from the definition of $\varepsilon(x)$, since as $x \rightarrow \infty$ holds (19) by Proposition 3 . In this sense we have

$$
\begin{gathered}
\frac{\gamma(x+\lambda) A(x+\lambda)-\gamma(x) A(x)}{A(x)}=\frac{f(x+\lambda)-f(x)}{A(x)}- \\
-\frac{1}{A(x)}\left(\int_{a}^{x+\lambda} h(t) A(t) d t-\int_{a}^{x} h(t) A(t) d t\right)= \\
=\frac{f(x+\lambda)-f(x)}{A(x)}-\frac{1}{A(x)}\left(\int_{a}^{x}[f(t+\lambda)-f(t)] d t-\int_{a-\lambda}^{a} f(t+\lambda) d t\right)
\end{gathered}
$$

hence, as $x \rightarrow \infty$, we obtain for each fixed $\lambda \geq 0$ that

$$
\frac{\gamma(x+\lambda) A(x+\lambda)-\gamma(x) A(x)}{A(x)} \rightarrow 0
$$


because as $x \rightarrow \infty$ we have that $(f(x+\lambda)-(f(x)) / A(x) \rightarrow R(\lambda), A(x) \rightarrow \infty$ and

$$
\frac{1}{A(x)} \int_{a}^{x}(f(t+\lambda)-f(t)) d t \rightarrow R(\lambda)
$$

also, by Proposition 3. On the other hand, from the equality (18) we have

$$
\frac{1}{x} \int_{a}^{x} f(y) d y=\int_{a}^{x} \gamma(t) A(t) d t
$$

so that, for any $\lambda \geq 0$, the following equality holds of the form

$$
\frac{1}{A(x)} \int_{x}^{x+\lambda} \gamma(t) A(t) d t=\frac{1}{A(x)}\left(\int_{a}^{x}(f(t+\lambda)-f(t)) d t\right)+o(1)
$$

as $x \rightarrow \infty$ as before, so that

$$
\frac{1}{A(x)} \int_{x}^{x+\lambda} \gamma(t) A(t) d t==R(\lambda)+o(1)
$$

as before. In the following putting $t=x+y$ such that as $x \rightarrow \infty$ we have

$$
\frac{1}{A(x)} \int_{x}^{\lambda} \gamma(x+y) A(x+y) d y=R(\lambda)+o(1)
$$

hence we obtain that

$$
\int_{0}^{\lambda} \frac{\gamma(x+y) A(x+y)-\gamma(x) A(x)}{A(x)} d y+\lambda \gamma(x)=R(\lambda)+o(1)
$$

as $x \rightarrow \infty$. Thus, so finally we see as $x \rightarrow \infty$ that is $\gamma(x) \rightarrow R(\lambda) / \lambda$. The proof is complete.

3. Translational $O$-regularly varying behaviour. Let $x \mapsto G(x)$ be a measurable positive function on $I_{a}$ for some $a>0$, and $f$ be a real valued function, defined for sufficiently large positive value of its argument such that the limit

$$
f^{*}(\lambda):=\limsup _{x \rightarrow \infty} \frac{f(x+\lambda)-f(x)}{G(x)} \text { or } f_{*}(\lambda):=\liminf _{x \rightarrow \infty} \frac{f(x+\lambda)-f(x)}{G(x)}
$$

exists for every $\lambda \geq 0$.

When $G \in \operatorname{Tr}(R V)$ we define $\operatorname{Tr}(E K B)$ as the class of measurable functions $f$ such that for some constants $\rho$ and $\tau$ we have

$$
\rho h_{\sigma}(\lambda) \leq f_{*}(\lambda) \leq f^{*}(\lambda) \leq \tau h_{\sigma}(\lambda)
$$

for every $\lambda \geq 0$, where $h_{\sigma}(\lambda) \equiv \lambda$ for $\sigma=0$ and $h_{\sigma}(\lambda) \equiv \sigma^{-1}\left(e^{\sigma \lambda}-1\right)$ for $\sigma \neq 0$.

Throughout this section, $\psi(\lambda)$ will denote the function

$$
\psi(\lambda):=\limsup _{x \rightarrow \infty} \sup _{\mu \in[0, \lambda]} \frac{f(x+\mu)-f(x)}{G(x)}
$$


and $\mathcal{D}(\lambda, \mu)$ will denote the function

$$
\mathcal{D}(\lambda, \mu):=\limsup _{x \rightarrow \infty} \sup _{\gamma \in[\lambda, \mu]} \frac{f(x+\gamma)-f(x)}{G(x)}
$$

for $\mu \geq \lambda \geq 0$.

In connection with this, the positive function $f$ has translational bounded increase (or $f \in \operatorname{Tr}(B I))$ if $\mathcal{A}(\lambda, f)<\infty$, where

$$
\mathcal{A}(\lambda, f):=\limsup _{x \rightarrow \infty} \sup _{\mu \in[0, \lambda]} \frac{f(x+\mu)}{f(x)} ;
$$

or equivalently, if $\operatorname{tr} \alpha(f)<\infty$, where $\operatorname{tr} \alpha(f)$ is the infimum of those $\alpha$ for which there exists a constant $C=C(\alpha)$ such that for each $q>0$ we have

$$
\frac{f(x+\lambda)}{f(x)} \leq C[1+o(1)] e^{\lambda \alpha} \quad(x \rightarrow \infty)
$$

uniformly in $\lambda \in[0, q]$.

Proposition 4. Assume $G$ has translational bounded increase, i.e., $G \in$ $\operatorname{Tr}(B I)$. If $\psi(\lambda)<\infty$ for some $\lambda>0$, then $\psi(\lambda)<\infty$ for every $\lambda>0$.

Proof. Let $G \in \operatorname{Tr}(B I)$. For $\lambda, \mu \geq 0$ we have from (5) the following essential equality of the form

$$
\begin{aligned}
\sup _{\gamma \in[\mu, \lambda+\mu]} \frac{f(x+\gamma)-f(x)}{G(x)} & =\frac{G(x+\mu)}{G(x)} \sup _{\gamma \in[0, \lambda]} \frac{f(x+\mu+\gamma)-f(x+\mu)}{G(x+\mu)}+ \\
& +\frac{f(x+\mu)-f(x)}{G(x)} ;
\end{aligned}
$$

and since the supremum on the right is nonegative and $G \in \operatorname{Tr}(B I)$ whence we obtain

$$
\mathcal{D}(\mu, \lambda+\mu) \leq C e^{\sigma \mu} \psi(\lambda)+\psi(\mu),
$$

i.e., since $\psi(\lambda+\mu)=\max \{\psi(\mu), \mathcal{D}(\mu, \lambda+\mu)\}$ we conclude

$$
\psi(\lambda+\mu) \leq C e^{\sigma \mu} \psi(\lambda)+\psi(\mu),
$$

hence, with monotonicity, we obtain the required result. The proof is complete.

In further, we consider the extent to which a statement such as $f^{*}(\lambda)<\infty$ is implicitly uniform in $\lambda$. The result below shows that some local uniformity is present if $f$ is measurable and the set of $\lambda$ for which $f^{*}(\lambda)<\infty$ is assumed is not too small.

Theorem 5. Let $G \in \operatorname{Tr}(B I)$ and let $f$ be a measurable function such that $f^{*}(\lambda)<\infty$ on a set in $[0, \infty)$ of positive measure. Then there exists $a_{0} \geq 0$ such that $f^{*}(\lambda)<\infty$ for all $\lambda \geq a_{0}$ and for every $[a, b]$ with $a_{0}<a<b<\infty$ is

$$
\limsup _{x \rightarrow \infty} \sup _{\lambda \in[a, b]} \frac{f(x+\lambda)-f(x)}{G(x)}<\infty .
$$


The proof of this statement is an analogous to the proof of Theorem 8 in Tasković [32, p. 117] and thus we omit it.

We notice that in Theorem 5 for $a>a_{0}$ and $\tau>\operatorname{tr} \alpha(G)$ there exist $x_{0}$ and $K$ depending on $a$ and $\tau$ such that with $\sigma=\max \{0, \tau\}$ is

$$
\frac{f(x+\lambda)-f(x)}{G(x)} \leq K e^{\sigma \lambda} \quad\left(\text { for } \lambda \geq a, x \geq x_{0}\right) .
$$

Theorem 6. Let $G \in \operatorname{Tr}(R V)$ and let $f$ be a measurable function such that $f^{*}(\lambda) \leq k(\lambda)$ on a set of positive measure in $\left[a_{0}, \infty\right)$ and

$$
k(\lambda+\mu) \geq e^{\sigma \mu} k(\lambda)+k(\mu)
$$

for $\lambda, \mu \geq a_{0} \geq a>0$, where $k$ is a measure function on $\left[a_{0}, \infty\right)$. Then there exists $b \geq a_{0}$ such that for all $\lambda \geq b$ is

$$
\frac{f(x+\lambda)-f(x)}{G(x)} \leq k(\lambda)+o(1) \text { as } x \rightarrow \infty ;
$$

and, further, for all $c$ and $d$ with $d>c \geq b$, the fact (25) holds uniformly for every $\lambda \in[c, d]$.

Proof. Let $G \in \operatorname{Tr}(R V)$. Then, from (5), we obtain the following inequality of the form

$$
f^{*}(\lambda+\mu) \leq e^{\sigma \mu} f^{*}(\lambda)+f^{*}(\mu) ;
$$

thus, together with (24), the set of $\lambda$ on which $f^{*}(\lambda) \leq k(\lambda)$ is closed under multiplication. Hence, from a result of Hille-Phillips [12], it follows (25).

Suppose that uniformity fails in (25). Then there exist $\varepsilon>0$ and the sequences $x_{n} \rightarrow \infty(n \rightarrow \infty)$ and $\lambda_{n} \in[c, d]$ such that

$$
\frac{f\left(x_{n}+\lambda_{n}\right)-f\left(x_{n}\right)}{G\left(x_{n}\right)}>k\left(\lambda_{n}\right)+\varepsilon
$$

for every $n \in \mathbb{N}$. Observe that there is a neighborhood $J_{n}$ of $\lambda_{n} \in[c, d]$ such that

$$
\frac{f\left(x_{n}+\lambda\right)-f\left(x_{n}\right)}{G\left(x_{n}\right)}>k(\lambda)+\varepsilon
$$

for any $\lambda \in J_{n}$. Define by induction a decreasing sequence of closed intervals $I_{k} \subset[c, d]$, and a subsequence $\left(x_{n(k)}\right)$ of $\left(x_{n}\right)$ such that

$$
\frac{f\left(x_{n(k)}+\mu\right)-f\left(x_{n(k)}\right)}{G\left(x_{n(k)}\right)} \geq k(\lambda)+\frac{\varepsilon}{3}
$$

for every $\mu \in I_{k}$. To define $I_{k+1}$ when $I_{k}$ is known, observe that if $\delta_{k}$ is the length of $I_{k}$, and $q$ an integer such that $q \delta_{k}>d-c$, then

$$
\frac{f\left(x+\delta_{k}\right)-f(x)}{G(x)} \leq k(\lambda)+\frac{\varepsilon}{3 q}
$$

as soon as $x$ is large enough. Hence we have a contradiction which completes the proof. 
Theorem 7. Let $G \in \operatorname{Tr}(R V)$ and $f \in \operatorname{Tr}(E K B)$, then (E) holds locally uniformly in $I_{a}$, i.e., for every $\mu>a>0$ we have

$$
\rho h_{\sigma}(\lambda)+o(1) \leq \frac{f(x+\lambda)-f(x)}{G(x)} \leq \tau h_{\sigma}(\lambda)+o(1)
$$

as $x \rightarrow \infty$ and uniformly in $\lambda \in[a, \mu]$.

The proof of this statement is a totally analogous with the preceding proof of Theorem 6 and thus we omit it.

The following result for translational rapid variation is an analogous with the preceding statements for uniformity.

Theorem 8. Let $f$ be a measurable function and let

$$
\frac{f(x+\lambda)-f(x)}{G(x)} \rightarrow+\infty
$$

an a set of positive measure in $I_{a}$ for $a>0$. Then there exists $b \geq a$ such that

$$
\lim _{x \rightarrow \infty} \frac{f(x+\lambda)-f(x)}{G(x)}=+\infty \quad(\lambda \geq b)
$$

uniformly in $\lambda$ over every interval $(d, \infty)$ for $d \geq b$. Further, $f$ is bounded on every finite interval sufficiently for to the right.

In this part, in connection with the preceding and former facts we define two pairs of indices in the following sense.

Here as before indices may be infinite; all other constants are finite; $\sup \varnothing:=-\infty$, inf $\varnothing:=+\infty ;$ and

$$
h(\lambda) \equiv h_{\sigma}(\lambda):=\int_{0}^{\lambda} e^{\sigma t} d t .
$$

The translational upper local $G$-index of $f$, denoted by $\operatorname{tr}\left(\tau_{f}\right)$, is the infimum of those $\tau$ for which, for every $a>0$,

$$
\frac{f(x+\lambda)-f(x)}{G(x)} \leq \tau h_{\sigma}(\lambda)+o(1)
$$

as $x \rightarrow \infty$ and uniformly in $\lambda \in[0, a]$.

On the other hand, the translational lower local $G$-index of $f$, denoted by $\operatorname{tr}\left(d_{f}\right)$, is the supremum of those $d$ for which, for every $a>0$,

$$
\frac{f(x+\lambda)-f(x)}{G(x)} \geq d h_{\sigma}(\lambda)+o(1)
$$

as $x \rightarrow \infty$ and uniformly in $\lambda \in[0, a]$.

It is easy to see that if $\operatorname{tr}\left(\tau_{f}\right)$ is finite we can put $\tau=\operatorname{tr}\left(\tau_{f}\right)$ in (26); similarly for $\operatorname{tr}\left(d_{f}\right)$ in (27). Also, Theorem 7 gives immediately the following result. 
Theorem 9. (Indices Theorem). A function $f \in \operatorname{Tr}(E K B)$ if and only if it is measurable and its translational local $G$-indices $\operatorname{tr}\left(\tau_{f}\right)$ and $\operatorname{tr}\left(d_{f}\right)$ are both finite.

We notice that we turn now to expressions for $\operatorname{tr}\left(\tau_{f}\right)$ in terms of the functions $f^{*}$ and $\psi$ defined in the preceding forms.

Proposition 5. If $\psi(0+)>0$, then the translational upper local $G$-index of $f \operatorname{tr}\left(\tau_{f}\right)=+\infty$. If $\psi(0+)=0$, then there exists

$$
\operatorname{tr}\left(\tau_{f}\right)=\lim _{\lambda \downarrow 0} \frac{f^{*}(\lambda)}{e^{\lambda}-1}=\lim _{\lambda \downarrow 0} \frac{f^{*}(\lambda)}{h(\lambda)}=\sup _{\lambda>0} \frac{f^{*}(\lambda)}{h(\lambda)} \in[-\infty, \infty] .
$$

In connection with this equalities, we notice that the following equalities hold in the form as

$$
\lim _{\lambda \downarrow 0} \frac{\psi(\lambda)}{e^{\lambda}-1}=\lim _{\lambda \downarrow 0} \frac{\psi(\lambda)}{h(\lambda)}=\sup _{\lambda>0} \frac{\psi(\lambda)}{h(\lambda)} \in[0, \infty] .
$$

In connection with the preceding, for $G \equiv A \in \operatorname{Tr}(S V)$, the translational upper global $A$-index of $f$, denoted by $\operatorname{tr}\left(\alpha_{f}\right)$, is the infimum of those $\alpha$ for which there exists a constant $C=C(\alpha)$ such that, for every $a>0$,

$$
\frac{f(x+\lambda)-f(x)}{A(x)} \leq C+\alpha \lambda+o(1)
$$

as $x \rightarrow \infty$ and uniformly in $\lambda \in[0, a]$.

Also, the translational lower global $A$-index of $f$, denoted by $\operatorname{tr}\left(\beta_{f}\right)$, is the supremum of those $\beta$ for which there exists a constant $m=m(\beta)$ such that, for every $a>0$,

$$
\frac{f(x+\lambda)-f(x)}{A(x)} \geq m+\beta \lambda+o(1)
$$

as $x \rightarrow \infty$ and uniformly in $\lambda \in[0, a]$.

In further, for $G \in \operatorname{Tr}(B I)$, the class $\operatorname{Tr}(O K B)$ is the class of measurable $f$ that satisfy

$$
f(x+\lambda)-f(x)=O(G(x)), \quad \text { as } x \rightarrow \infty,
$$

for every $\lambda \geq 0$; and $\operatorname{Tr}(o K B)$ is the class of measurable $f$ that satisfy

$$
f(x+\lambda)-f(x)=o(G(x)), \quad \text { as } x \rightarrow \infty,
$$

for every $\lambda \geq 0$.

Unlike the translational local indices of the preceding part, the translational global indices are defined only for $\sigma=0$. However for calculating the indices it is desirable to have expressions in terms of the functions $f^{*}$ and $\psi$ under suitable conditions. 
Proposition 6. If $\psi<\infty$, then there exists the translational upper global $A$-index of $f$ in the following form

$$
\operatorname{tr}\left(\alpha_{f}\right)=\lim _{\lambda \rightarrow \infty} \frac{f^{*}(\lambda)}{\lambda}=\inf _{\lambda>0} \frac{f^{*}(\lambda)}{\lambda} \in[-\infty, \infty] .
$$

In connection with this equalities, we notice that there exists

$$
\lim _{\lambda \rightarrow \infty} \frac{\psi(\lambda)}{\lambda}=\inf _{\lambda>0} \frac{\psi(\lambda)}{\lambda} \in[-\infty, \infty] .
$$

Now, Theorem 3 gives immediately the following result as an Global Indices Theorem.

Theorem 10. Let $A \in \operatorname{Tr}(S V)$ and $f$ be a measurable function. Then, a function $f \in \operatorname{Tr}(O K B)$ if and only if its translational global indices $\operatorname{tr}\left(\alpha_{f}\right)$ and $\operatorname{tr}\left(\beta_{f}\right)$ are both finite.

In further, we first give the basic representations for the classes $\operatorname{Tr}(O K B)$ and $\operatorname{Tr}(E K B)$.

Theorem 11. (Representation for $\operatorname{Tr}(O K B)$ ). Let $G \in \operatorname{Tr}(B I)$ be a measurable function. Then $f \in \operatorname{Tr}(O K B)$ if and only if

$$
f(x)=C+\eta(x) G(x)+\int_{b}^{x} \xi(t) G(t) d t
$$

(i.e., if and only if there exists $\sigma \in \mathbb{R}$ such that

$$
\left.f(x)=C+\sigma x+\eta(x) G(x)+\int_{b}^{x} \xi(t) G(t) d t\right)
$$

for $x \geq b$, where $C$ and $b$ are constants and the measurable functions $\eta$ and $\xi$ are bounded.

Proof. If $f$ is a function given by (28), then we obtain following inequality in the form

$$
\frac{|f(x+\lambda)-f(x)|}{G(x)} \leq \delta(x)\left[1+\frac{G(x+\lambda)}{G(x)}+\int_{0}^{\lambda} \frac{G(x+u)}{G(x)} d t\right]
$$

for $\lambda>0$ and $x \geq b$, where $\delta(x)=\max \left\{\sup _{[x, \infty]} \eta, \sup _{[x, \infty)} \xi\right\}$. This means, since $T \in \operatorname{Tr}(B I)$, boundedness as $x \rightarrow \infty$, so $f \in \operatorname{Tr}(O K B)$. For the converse write

$f(x)=\int_{b}^{b+s} f(t) d t+G(x) \int_{x}^{x+s} \frac{f(x)-f(t)}{G(x)} d t+\int_{b}^{x} \frac{f(t+s)-f(t)}{G(t)} G(t) d t$,

for large enough $b$. By Theorem 3 the term $(f(x)-f(t)) / G(x)$ in the second integral is uniformly bounded for large $x$, so the middle term on the right is $O(G(x))$. In the third integral the term $(f(t+s)-f(t)) / G(t)$ is $O(1)$ and the first term on the right is constant. The proof is complete. 
Remarks. The statement reamains true if $f \in \operatorname{Tr}(O K B)$ is replaced by $f \in \operatorname{Tr}(o K B)$, where bounded means $o(1)$ as $x \rightarrow \infty$. Also, measurability of $G$ is only a convenience here. If $G$ is not measurable, replace $\xi(t) G(t)$ in $(28)$ or $(29)$ by $\mathcal{F}(t)$, where $\mathcal{F}(t)$ is a measurable function and $O(G)$ or $o(G)$. Then the representation still is valid.

Theorem 12. (Representation Theorem for $\operatorname{Tr}(E K B))$. Let $G \in \operatorname{Tr}(R V)$. Then $f \in \operatorname{Tr}(E K B)$ if and only if

$$
f(x)=C+\eta(x) G(x)+\int_{b}^{x} \xi(t) G(t) d t
$$

(i.e., if and only if there exists $\sigma \in \mathbb{R}$ such that

$$
\left.f(x)=C+\sigma x+\eta(x) G(x)+\int_{b}^{x} \xi(t) G(t) d t\right)
$$

for $x \geq b$, where $C$ and $b$ are constants, $\eta(x) \rightarrow 0$ as $x \rightarrow \infty$, $\xi$ is bounded, and both $\eta$ and $\xi$ are mesaurable. The translational local indices of $f$ are given by

$$
\begin{aligned}
& \operatorname{tr}\left(\tau_{f}\right)=\lim _{\lambda \downarrow 0} \limsup _{x \rightarrow \infty} \frac{1}{e^{\lambda}-1} \int_{x}^{x+\lambda} \xi(t) d t, \\
& \operatorname{tr}\left(d_{f}\right)=\lim _{\lambda \downarrow 0} \liminf _{x \rightarrow \infty} \frac{1}{e^{\lambda}-1} \int_{x}^{x+\lambda} \xi(t) d t,
\end{aligned}
$$

and satisfy the following inequalities of the form

$$
\operatorname{tr}\left(\tau_{f}\right) \leq \limsup _{t \rightarrow \infty} \xi(t) \quad \text { and } \quad \operatorname{tr}\left(d_{f}\right) \geq \liminf _{t \rightarrow \infty} \xi(t) .
$$

The proof of this statement is an analogous with the preceding proof of Theorem 11 and thus we omit it.

4. Some open problems. We shall say that a positive, finite and measurable function $f$, denoted on $I_{a}$ for some $a>0$, is a translational homothetic function at infinity (denoted this class by $T H$ ) if the limit

$$
\lim _{x \rightarrow \infty} \frac{f(\lambda x+\tau)}{f(x)}=k(\lambda, \tau)
$$

is positive and finite for all $\lambda>0$ and $\tau \geq 0$. Thus, from (29a), for $\lambda=1$ we obtain the class $\operatorname{Tr}(R V)$ and for $\tau=0$ we have the class $R V$ in Karamata's sense.

In connection with the preceding, we can considered an analogous translational homothetic behaviour in the following sense as the limit

$$
\lim _{x \rightarrow \infty} \frac{f(\lambda x+\tau)-f(x)}{G(x)}=h(\lambda, \tau) \in \mathbb{R} \cup\{\infty\}
$$


for all $\lambda>0$ and all $\tau \geq 0$, where $G: I_{a} \rightarrow I_{a}$ for some $a>0$. For $\lambda=1$, from (30), we obtain the class $\operatorname{Tr}(K B)$ and for $\tau=0$ we have the class $K B$ in Bojanić - Karamata's sense.

The class of all functions $f$ satisfying relation (30) is denoted by $\operatorname{Ex}(T H)$.

Open problem 1. To invent representation of the class Ex $(T H)$ of the all extension translational homothetic functions!? Also, to invent and some characterizations of the class functions $\operatorname{Ex}(T H)$ !?

We notice that parallel to the $E x(T H)$ functions we can consider $E x(O T H)$ functions as an analogous behaviour with the class $O T H$ functions introduced in Tasković [32]. In this sense we can consider the following limit

$$
\limsup _{x \rightarrow \infty} \frac{f(\lambda x+\tau)-f(x)}{G(x)}=k(\lambda, \tau) \in \mathbb{R} \cup\{\infty\}
$$

for all $\lambda>0$ and all $\tau \geq 0$, where $G: I_{a} \rightarrow I_{a}$ for some $a>0$. Thus, from (31), for $\lambda=1$ we obtain the class $\operatorname{Tr}(O K B)$ and for $\tau=0$ we have the class $O K B$ functions which are study was initiaeted by Bojanić and Karamata [6], and indipendently by L. de Haan [10].

The class of all functions $f$ satisfying relation (31) is denoted by $\operatorname{Ex}(O T H)$.

Open problem 2. To invent representation of the class Ex $(O T H)$ of the all extension $O$-translational homothetic functions!? Also, to invent and some characterizations of the class functions $\operatorname{Ex}(O T H)$ !?

5. Tauberian Nature Theorems. The theorems which we shall treat first are called Abelian because they are generalization of a familiar result of Abel. It was A. Tauber in 1897 year who first gave a conditional converse of Abel's theorem.

Tauberian statements are concerned with the deduction of the asymptotic behaviour of functions from the asymptotic behaviour of their transforms. In this sense, first, essential result is a very useful theorem due to Hardy and Littlewood in 1912 year in its original form.

In connection with this, well known an integral extension results of Hardy and Littlewood which due J. Karamata in 1931 year with an ingenious proof via the regularly varying functions.

In this section we give some results of Tauberian nature in support of the Karamata's lemma (see: [18, Hauptsatz 1]), via translational regularly varying functions.

We notice that first fundamental result of Tauberian nature for Laplace Stieltjes transform in 1929 due to Hardy and Littlewood [11] in the following form.

Theorem HL. Let $t \mapsto f(t) \geq 0$ for $t>0$ and $t \mapsto e^{-s t} f(t)$ is an integrable function in $(0, \infty)$ such that

$$
\int_{0}^{\infty} e^{-s t} f(t) d t \sim C s^{-\delta} \quad(C \geq 0, \quad \delta>0)
$$


as $s \rightarrow 0$, then

$$
\int_{0}^{x} f(t) d t \sim \frac{C}{\Gamma(\delta+1)} x^{\delta} \quad \text { as } \quad x \mapsto \infty
$$

For this and some other results in 1931 Karamata give further extensions and new facts well known as Karamata's Tauberian theorems. In this sense the following initial result due to Karamata [18].

Theorem K. Let $t \mapsto \alpha(t)$ be nondecreasing function and such that the following integral of the form

$$
f(s)=\int_{0}^{\infty} e^{-s t} d(\alpha(t))
$$

converges for $s>0$ and for some positive number $\gamma>0$ let

$$
f(s) \sim \frac{1}{s^{\gamma}} \quad(\text { as } s \rightarrow 0 \text { or }+\infty)
$$

and let $x \mapsto g(x)$ be of bounded variation in $(0,1)$. Then the following relation holds

$$
\int_{0}^{\infty} e^{-s t} g\left(e^{-s t}\right) d(\alpha(t)) \sim \frac{1}{s^{\gamma} \Gamma(\gamma)} \int_{0}^{\infty} e^{-t} g\left(e^{-t}\right) t^{\gamma-1} d t
$$

as $s \rightarrow 0$ or $+\infty$, where $s$ varying through the set of points for which the integral the left exists.

It was Littlewood in 1910 who first showed that the condition $a_{n}=o\left(n^{-1}\right)$ in a Tauber's theorem could be replaced by the condition $a_{n}=O\left(n^{-1}\right)$. In this sense and connection with the results of Karamata [17] and [18] we begin with the following statement.

Theorem 13. Let $t \mapsto \alpha(t)$ be nondecreasing function and such that the following integral of the form

$$
f(s)=\int_{0}^{\infty} e^{-s t} d(\alpha(t))
$$

converges for $s>0$ and for $n \in \mathbb{N}:=\{1,2,3, \ldots\}$ and for some positive number $\rho$ let the following asymptotic relation holds

$$
f(s) \sim \exp \left( \pm \frac{\rho s}{n+1}\right) A\left(\frac{s}{n+1}+n\right) \quad \text { as } s \rightarrow \infty,
$$

where $A \in \operatorname{Tr}(S V)$. If $x \mapsto g(x)$ is a bounded variation function in $(0,1)$, then

$$
\int_{0}^{\infty} e^{-s t} g\left(e^{-s t}\right) d(\alpha(t)) \sim \frac{e^{ \pm \rho s} A(s)(n+1)^{\rho}}{\Gamma(\rho)} \int_{0}^{\infty} e^{-t} g\left(e^{-t}\right) t^{\rho-1} d t
$$

as $s \rightarrow \infty$, where $s$ varying through the set of points for which the integral on the left exists. 
Proof. Let $\alpha(t)$ be continuous except perhaps in the set of points $x_{0}, x_{1}, x_{2}, \ldots$ and let $g\left(e^{-t}\right)$ be continuous except perhaps in the points $y_{0}, y_{1}, y_{2}, \ldots$; and denote by $S$ the set of points $y_{i} / x_{i}(i, j=0,1,2, \ldots)$. If $s$ is not in $S$ the integral on the left of (32) exists since $\alpha(t)$ and $g\left(e^{-s t}\right)$ will have no common discontinuities.

Since $S$ is a countable set its complement in dense in $(0, \infty)$ so that $s$ may approach zero or become infinite while remaining in the set of points for which the integral on the left of (33) exists.

Let $\varepsilon$ be an arbitrary positive number. Determine polynomials $p(x)$ and $P(x)$, from Karamata [18, Hauptsatz 1] so that

$$
p(x)<g(x)<P(x) \text { for } 0 \leq x \leq 1,
$$

and

$$
\frac{1}{\Gamma(\rho)} \int_{0}^{\infty} e^{-t} t^{\rho-1}\left[P\left(e^{-t}\right)-p\left(e^{-t}\right)\right] d t<\varepsilon ;
$$

thus, since $t \mapsto \alpha(t)$ is nondecreasing, we obtain the following inequalities of the form

$\int_{0}^{\infty} e^{-s t} p\left(e^{-s t}\right) d(\alpha(t)) \leq \int_{0}^{\infty} e^{-s t} g\left(e^{-s t}\right) d(\alpha(t)) \leq \int_{0}^{\infty} e^{-s t} P\left(e^{-s t}\right) d(\alpha(t))$

and

$$
\begin{gathered}
\frac{(n+1)^{\rho}}{\Gamma(\rho)} \int_{0}^{\infty} e^{-t} p\left(e^{-t}\right) t^{\rho-1} d t \leq \frac{(n+1)^{\rho}}{\Gamma(\rho)} \int_{0}^{\infty} e^{-t} g\left(e^{-t}\right) t^{\rho-1} d t \leq \\
\leq \frac{(n+1)^{\rho}}{\Gamma(\rho)} \int_{0}^{\infty} e^{-t} P\left(e^{-t}\right) t^{\rho-1} d t
\end{gathered}
$$

such that, if we replace $s$ by $(n+1) s$ in the relation (32) we obtain

$$
\int_{0}^{\infty} e^{-s t} e^{-n s t} d(\alpha(t)) \sim e^{ \pm \rho s} A(s+n)=\frac{e^{ \pm \rho s} A(s+n)}{(n+1)^{-\rho} \Gamma(\rho)} \int_{0}^{\infty} e^{-t} e^{-n t} t^{\rho-1} d t
$$

for any positive integer $n$, whence we have the following asymptotic relations of the form

$$
\begin{gathered}
\int_{0}^{\infty} e^{-s t} P\left(e^{-s t}\right) d(\alpha(t)) \sim \frac{e^{ \pm \rho s} A(s+n)}{(n+1)^{-\rho} \Gamma(\rho)} \int_{0}^{\infty} e^{-t} P\left(e^{-t}\right) t^{\rho-1} d t= \\
=\frac{e^{ \pm \rho s} A(s+n)(n+1)^{\rho} A(s)}{A(s) \Gamma(\rho)} \int_{0}^{\infty} e^{-t} P\left(e^{-t}\right) t^{\rho-1} d t \sim \\
\sim \frac{e^{ \pm \rho s} A(s)(n+1)^{\rho}}{\Gamma(\rho)} \int_{0}^{\infty} e^{-t} P\left(e^{-t}\right) t^{\rho-1} d t
\end{gathered}
$$


for any polynomial $P(x)$ and any function $A \in \operatorname{Tr}(S V)$. On the other hand, from (35), we have

$$
\begin{array}{r}
\quad \frac{(n+1)^{\rho}}{\Gamma(\rho)} \int_{0}^{\infty} e^{-t} p\left(e^{-t}\right) t^{\rho-1} \leq \liminf _{s \rightarrow \infty} \frac{e^{\mp \rho s}}{A(s)} \int_{0}^{\infty} e^{-s t} g\left(e^{-s t}\right) d(\alpha(t)) \leq \\
\leq \limsup _{s \rightarrow \infty} \frac{e^{\mp \rho s}}{A(s)} \int_{0}^{\infty} e^{-s t} g\left(e^{-s t}\right) d(\alpha(t)) \leq \frac{(n+1)^{\rho}}{\Gamma(\rho)} \int_{0}^{\infty} e^{-t} P\left(e^{-t}\right) t^{\rho-1} d t,
\end{array}
$$

where $s$ approaching infinite in the set complementary to $S$. From (34), (36) and (37) we see that

$$
\lim _{s \rightarrow \infty} \frac{e^{\mp \rho s}}{A(s)} \int_{0}^{\infty} e^{-s t} g\left(e^{-s t}\right) d(\alpha(t))=\frac{(n+1)^{\rho}}{\Gamma(\rho)} \int_{0}^{\infty} e^{-t} g\left(e^{-t}\right) t^{\rho-1} d t,
$$

the desired result. The proof is complete.

From the preceding result of Theorem 13 by specializing $g(x)$ we may obtain the following form of Tauberian statement.

Theorem 14. Let $t \mapsto \alpha(t)$ be nondecreasing function and such that the following integral of the form

$$
f(s)=\int_{0}^{\infty} e^{-s t} d(\alpha(t))
$$

converges for $s>0$ and for $n \in \mathbb{N}$ and for some positive number $\rho$ let the following asymptotic relation holds

$$
f(s) \sim \exp \left( \pm \frac{\rho s}{n+1}\right) A\left(\frac{s}{n+1}+n\right) \quad \text { as } s \rightarrow \infty,
$$

where $A \in \operatorname{Tr}(S V)$, then

$$
\alpha(t) \sim \frac{e^{ \pm \rho t} A(t)(n+1)^{\rho}}{\Gamma(\rho+1)} \text { as } t \rightarrow+\infty .
$$

Proof. (Application of Theorem 13). If $\rho>0$ we choose the function $g\left(e^{-t}\right)$ of Theorem 13 as follows:

$$
g\left(e^{-t}\right)= \begin{cases}e^{t} & \text { for } \quad 0 \leq t \leq 1 \\ 0 & \text { for } \quad 1<t<\infty\end{cases}
$$

then the conclusion of Theorem 13 is preciesely (38) provided $t$ approaches its limit through the set of points where $\alpha(t)$ is continuous. Obviously this restriction may be removed since $\alpha(t)$ is monotonic. The proof is complete.

In connection with the preceding facts we can prove the following statement which is an extension of Theorem 13.

Theorem 15. Let $t \mapsto \alpha(t)$ be nondecreasing function and such that the following integral of the form

$$
f(s)=\int_{0}^{\infty} e^{-s t} d(\alpha(t))
$$


converges for $s>0$ and for $n \in \mathbb{N}$ and for some positive numbers $\rho$ and $\delta$ let the following asymptotic relation holds

$$
f(s) \sim s^{-\delta} \exp \left( \pm \frac{\rho s}{n+1}\right) A\left(\frac{s}{n+1}+n\right) \quad \text { as } s \rightarrow \infty,
$$

where $A \in \operatorname{Tr}(S V)$. If $x \mapsto g(x)$ is a bounded variation function in $(0,1)$, then

$$
\int_{0}^{\infty} e^{-s t} g\left(e^{-s t}\right) d(\alpha(t)) \sim \frac{e^{ \pm \rho s} A(s)}{s^{\delta} \Gamma(\delta)} \int_{0}^{\infty} e^{-t} g\left(e^{-t}\right) t^{\delta-1} d t
$$

as $s \rightarrow \infty$, where $s$ varying through the set of points for which the integral on the left exists.

The proof of this statement is a totally analogous with the preceding proof of Theorem 13, and thus we omit it.

Similar to the system for Theorem 14, from the preceding result of Theorem 15 , specializing $g(x)$ with (39) we may directly obtain the following form of Tauberian statement.

Theorem 16. Let $t \mapsto \alpha(t)$ be nondecreasing function and such that the following integral of the form

$$
f(s)=\int_{0}^{\infty} e^{-s t} d(\alpha(t))
$$

converges for $s>0$ and for $n \in \mathbb{N}$ and for some positive numbers $\rho$ and $\delta$ let the following asymptotic relation holds

$$
f(s) \sim s^{-\delta} \exp \left( \pm \frac{\rho s}{n+1}\right) A\left(\frac{s}{n+1}+n\right) \quad \text { as } s \rightarrow \infty,
$$

where $A \in \operatorname{Tr}(S V)$, then

$$
\alpha(t) \sim \frac{t^{-\delta} e^{ \pm \rho t} A(t)}{\Gamma(\delta+1)} \quad \text { as } t \rightarrow \infty .
$$

6. Monotone Density Statements. In this section we give only a simplest Tauberian theorem to show the flavour of the arguments. In this sense, a typical result of the Tauberian nature can be stated as follows.

Theorem 17. Let $x \mapsto G(x)$, defined and positive on $I_{a}:=[a, \infty)$ for some $a>0$, be given by

$$
G(x)=\int_{b}^{x} k(t) d t \quad \text { for } \quad b \geq a,
$$

where $t \mapsto k(t)$ is ultimately monotone (i.e., monotone for $t$ sufficiently enough). Then for $\sigma \geq 0$ we have that

$$
G(x)=e^{\sigma x} A(x) \text { implies } \frac{k(x)}{G(x)} \rightarrow \sigma \quad(\text { as } x \rightarrow \infty),
$$

where $x \mapsto A(x)$ is an arbitrary translational slowly varying function. 
Proof. Suppose first that $k(t)$ is ultimately nondecreasing. Then for $\beta>\alpha \geq \alpha_{0}$ say,

$$
\frac{G(t+\beta)-G(t+\alpha)}{G(t)}=\int_{t+\alpha}^{t+\beta} \frac{k(y)}{G(t)} d y
$$

so that

$$
\frac{(\beta-\alpha) k(t+\alpha)}{G(t)} \leq \frac{G(t+\beta)-G(t+\alpha)}{G(t)} \leq \frac{(\beta-\alpha) k(t+\beta)}{G(t)} ;
$$

thus, letting $t \rightarrow \infty$, from the left hand side of

$$
\limsup _{t \rightarrow \infty} \frac{k(t+\alpha)}{G(t)} \leq \frac{e^{\sigma \beta}-e^{\sigma \alpha}}{\beta-\alpha},
$$

and letting $\beta \rightarrow \alpha$, since the left hand side of the present inequality is independent of $\beta$ we get

$$
\limsup _{t \rightarrow \infty} \frac{k(t+\alpha)}{G(t)} \leq \sigma e^{\sigma \alpha} .
$$

On the other hand, similarly, from the right hand side of (40) we obtain the following inequality

$$
\liminf _{t \rightarrow \infty} \frac{k(t+\beta)}{G(t)} \geq \sigma e^{\sigma \beta}
$$

and thus, since $\alpha$ and $\beta$ are arbitrary, for arbitrary $\gamma \geq \alpha_{0}$, we obtain the following equality

$$
\lim _{t \rightarrow \infty} \frac{k(t+\gamma)}{G(t)}=\sigma e^{\sigma \gamma},
$$

and so, putting $x=t+\gamma$ and using $G(t)=e^{\sigma t} A(t)$ completes the assertion. The second case where $k(t)$ is ultimately nonincreasing may be proved similarly. The proof is complete.

Annotation. We notice that the statement of Theorem 17 can extend to the case when

$$
G(x)=\int_{b}^{x} k(t) d(B(t)) \quad \text { for } \quad b \geq a,
$$

where $B(t)=e^{\sigma t} \mathcal{A}(t)$ is monotone nondecreasing and translational regularly varying with index $\sigma$ and $G(x)=e^{\rho x} A(x)$ for $\rho \geq 0$ and $A \in \operatorname{Tr}(S V)$ !

7. Stieltjes transform. We now prove a Tauberian theorem of a very special nature for Laplace-Stieltjes transform, i.e., for Stieltjes transform.

Theorem 18. If $t \mapsto U(t)$ is a nondecreasing function, $U\left(0_{-}\right)=0$ and $\rho>0$, let $S_{\rho}(U ; \cdot)$ be the Stieltjes transform of order $\rho$ such that

$$
S_{\rho}(U ; x):=\int_{0}^{\infty} \frac{d(U(t))}{(t+x)^{\rho}} \sim x^{-\delta} \exp \left( \pm \frac{\sigma x}{n+1}\right) A\left(\frac{x}{n+1}+n\right)
$$


as $x \rightarrow \infty$ for $\rho \geq \sigma>0, n \in \mathbb{N}, \delta>0, \rho+\delta>1$ and $A \in \operatorname{Tr}(S V)$, then for arbitrary $\gamma>0$ as $x \rightarrow \infty$ the following fact holds

$$
U(x) \sim \frac{e^{\rho \gamma} \Gamma(\rho+1) x^{1-\rho-\delta} e^{ \pm \sigma x} A(x)}{\Gamma(\delta+1) \Gamma(\rho+\delta)} .
$$

Proof. (Applications Theorem 16 and the method of Theorem 17). Since for $\rho>0$ we have the following equality of the form

$$
\frac{1}{(t+x)^{\rho}}=\frac{1}{\Gamma(\rho)} \int_{0}^{\infty} e^{-t \tau} e^{-x \tau} \tau^{\rho-1} d \tau,
$$

it follows that

$$
S_{\rho}(U ; x)=\int_{0}^{\infty} \frac{d(U(t))}{(t+x)^{\rho}}=\int_{0}^{\infty} g(\tau) e^{-x \tau} d \tau,
$$

where

$$
g(\tau)=\frac{\tau^{\rho-1}}{\Gamma(\rho)} \int_{0}^{\infty} e^{-t \tau} d(U(t)) ;
$$

and thus, from the given, and by use of Theorem 16 it follows that

$$
\int_{0}^{x} g(\tau) d \tau \sim \frac{x^{-\delta} e^{ \pm \sigma x} A(x)}{\Gamma(\delta+1)}
$$

as $x \rightarrow \infty$. Writing $g(\tau)$ out in full, we obtain the following asymptotic relation

$$
\frac{1}{\Gamma(\rho+1)} \int_{0}^{x} f(\tau) d\left(\tau^{\rho}\right) \sim \frac{x^{-\delta} e^{ \pm \sigma x} A(x)}{\Gamma(\delta+1)},
$$

where $f(\tau)=\int_{0}^{\infty} e^{-t \tau} d(U(t))$, so that

$$
\alpha(x):=\int_{0}^{x} f(\tau) d\left(\tau^{\rho}\right) \sim \frac{x^{-\delta} e^{ \pm \sigma x} A(x) \Gamma(\rho+1)}{\Gamma(\delta+1)}
$$

as $x \rightarrow \infty$, where $f(\tau)$, the Laplace-Stieltjes transform of $U(t)$ is monotone nonincreasing as $\tau$ increases. In further let $\beta>\alpha>0$. Then (similarly to the proof of Theorem 17) we have

$$
\frac{\alpha(x+\beta)-\alpha(x+\alpha)}{\alpha(x)}=\frac{\int_{x+\alpha}^{x+\beta} f(\tau) d\left(\tau^{\rho}\right)}{\alpha(x)} \geq \frac{f(x+\alpha)\left[(x+\beta)^{\rho}-(x+\alpha)^{\rho}\right]}{\alpha(x)},
$$

hence, using (41) and letting $x \rightarrow \infty$ we obtain the following inequality of the form

$$
\limsup _{x \rightarrow \infty} \frac{f(x+\alpha) x^{\rho-1}}{\alpha(x)} \leq e^{\rho \alpha}
$$

which holds true for arbitrary $\alpha>0$. On the other hand, similarly, we obtain

$$
\liminf _{x \rightarrow \infty} \frac{f(x+\beta) x^{\rho-1}}{\alpha(x)} \geq e^{\rho \beta}
$$


for arbitrary $\beta>0$. Hence, for arbitrary $\gamma>0$ we have the following fact of the form

$$
\lim _{x \rightarrow \infty} \frac{f(x+\gamma) x^{\rho-1}}{\alpha(x)} \leq e^{\rho \gamma}
$$

thus, putting $t=x+\gamma$, we obtain that

$$
f(t) \sim e^{\rho \gamma}(t-\gamma)^{1-\rho} \alpha(t-\gamma)
$$

as $t \rightarrow \infty$, i.e., from the preceding facts we have as $t \rightarrow \infty$ the following asymptotic relation

$$
f(t) \sim \frac{e^{\rho \gamma}(t-\gamma)^{1-\rho-\delta} e^{ \pm \sigma(t-\gamma)} A(t-\gamma) \Gamma(\rho+1)}{\Gamma(\delta+1)}
$$

taking into account (41). Since, in the general case, for $n \in \mathbb{N}$ and $y=t-\gamma$ we have

$$
y^{1-\rho-\delta} e^{ \pm \sigma y} A(y) \sim y^{1-\rho-\delta} \exp \left(\frac{ \pm \sigma y}{n+1}\right) A\left(\frac{y}{n+1}+n\right)
$$

as $y \rightarrow \infty$, thus applying now Theorem 16, from the preceding facts it follows that

$$
U(x) \sim \frac{e^{\rho \gamma} \Gamma(\rho+1)}{\Gamma(\delta+1)} \frac{x^{1-\rho-\delta} e^{ \pm \sigma x} A(x)}{\Gamma(\rho+1)}
$$

as $x \rightarrow \infty$, which is the required result. The proof is complete.

Annotation. The preceding result of Theorem 18 is connection with the result of Karamata [18] and the classical statement in 1914 of Hardy and Littlewood.

8. Tauberian Theorem for Power Series. One may specialise Theorem 16 to Dirichlet series $\sum_{n=0}^{\infty} a_{n} \exp \left(-\lambda_{n} s\right)$. The case $\lambda_{n}=n$ is particularly important; writing $s$ in place of $e^{-s}$ one studies the power series

$$
f(s)=\sum_{n=0}^{\infty} a_{n} s^{n},
$$

the results above yield as a directly consequence of the preceding Tauberian nature statements.

Theorem 19. If $a_{n} \geq 0$ for $n \in \mathbb{N} \cup\{0\}$ and the power series (43) converges for $s \in[0,1)$, then the asymptotic relation of the form

$$
f(s) \sim(1-s)^{\delta} \exp \left(\frac{\sigma}{(1-s)(k+1)}\right) A\left(\frac{1}{(1-s)(k+1)}+k\right)
$$

as $s \rightarrow 1-$, for $\delta, \sigma \geq 0, k \in \mathbb{N}$ and $A \in \operatorname{Tr}(S V)$, implies

$$
\sum_{k=0}^{n-1} a_{k} \sim \frac{n^{-\delta} e^{\sigma n} A(n)}{\Gamma(\delta+1)} \quad \text { as } n \rightarrow \infty .
$$


Proof. Defining

$$
\alpha(x)=\int_{0}^{x} u(t) d t, \quad \text { for } \quad x \geq 0
$$

where $u(t)=a_{n}$ for $n \leq t<n+1$ and for $n \in \mathbb{N} \cup\{0\}$. Then we have that

$$
\alpha(x)=\sum_{k=1}^{n-1} a_{k}+a_{n}(x-n)
$$

is a continuous function with properties $\alpha(0)=0$ and $\alpha(n)=\sum_{k=0}^{n-1} a_{k}$. Defining, in further, that is

$$
h(s)=\int_{0}^{\infty} e^{-s t} d(\alpha(t))
$$

we obtain

$$
\begin{gathered}
h(s)=\sum_{n=0}^{\infty} \int_{n}^{n+1} e^{-s t} d(\alpha(t))=\sum_{n=0}^{\infty} \int_{0}^{1} e^{-s(n+t)} d(\alpha(n+t))= \\
=\sum_{n=0}^{\infty} e^{-s n} a_{n}\left(\int_{0}^{1} e^{-s t} d t\right)=\frac{1-e^{-s}}{s} \sum_{n=0}^{\infty} a_{n} e^{-s n}=\frac{1-e^{-s}}{s} f\left(e^{-s}\right),
\end{gathered}
$$

hence, by (44), as $s \rightarrow 0+$ we have

$h(s) \sim \frac{1-e^{-s}}{s}\left(1-e^{-s}\right)^{\delta} \exp \left(\frac{\sigma}{\left(1-e^{-s}\right)(k+1)}\right) A\left(\frac{1}{\left(1-e^{-s}\right)(k+1)}+k\right)$, i.e.,

$$
h(s) \sim s^{-\delta} \exp \left(\frac{\sigma s}{k+1}\right) A\left(\frac{s}{k+1}+k\right) \quad \text { as } \quad s \rightarrow \infty .
$$

Applying Theorem 16, directly, we obtain the following asymptotic relation in the following form

$$
\alpha(t) \sim \frac{t^{-\delta} e^{\sigma t} A(t)}{\Gamma(\delta+1)} \quad \text { as } \quad t \rightarrow \infty
$$

i.e., thus

$$
\alpha(n):=\sum_{k=0}^{n-1} a_{k} \sim \frac{n^{-\delta} e^{\sigma n} A(n)}{\Gamma(\delta+1)} \quad \text { as } \quad n \rightarrow \infty .
$$

The proof is complete. (In connection with the preceding statement also see and a result by Djurčić [26]). 


\section{REFERENCES}

[1] J. M. Ash, P. Erdös, and L.A. Rubel: Very slowly varying functions, Aequationes Math., 10(1974), 1-9.

[2] N. H. Bingham and C.M. Goldie: Extensions of regular variation, I-Uniformity and Quantifiers, Proc. London Math. Soc. (3), 44 (1982), 473-496.

[3] N. H. Bingham and C.M. Goldie: Extensions of regular variation, II-Representations and indices, Proc. London Math. Soc. (3), 44 (1982), 497-534.

[4] N. H. Bingham, C.M. Goldie, and J.L. Teugels: Regular Variation, Cambridge Univ. Press, Cambridge, 1987.

[5] R. Bojanić, and E. Seneta: Slowly Varying Functions and Asymptotic Relations, J. Math. Anal. Appl., 34(1971), 302-315.

[6] R. Bojanić, and J. Karamata: On a Class of Functions of Regular Asymptotic Behaviour, Math. Res. Centre Tech. Summary Report 436. Madison: University of Wisconsin 1963.

[7] N. G. de Bruijn: Pairs of slowly oscillating functions occuring in asymptotic problems concerning the Laplace transform, Nieuw Arch.Wisk., 7(1959), 20-26.

[8] Csiszár and P. Erdös: On the function $g(t)=\lim _{\sup _{x \rightarrow \infty}}[f(x+t)-f(x)]$, Magyar Tud. Akad., Mat. Kut. Int. Közl., Ser. A, 9 (1964), 603-606.

[9] H. Delange: Sur un théorème de Karamata, Bull.Sci.Math. France, 79(1955), 9-12.

[10] L. de Haan: On regular Variation and Its Application to the Weak Convergence of Sample Extremes, Mathematical Centre Tracts 32, Amsterdam, 1970.

[11] G.H. Hardy, and J.E. Littlewood: Notes on the theory of series XI-On Tauberian theorems, Proc. London Math. Soc., 30 (1929), 23-37.

[12] E. Hille and R.S.Phillips: Functional analysis and semigroups, revised edn. American Mathematical Society Colloq. Publ., Providence, R.I., 1957.

[13] J. Karamata: Sur certains "Tauberian theorems" de M.M. Hardy et Littlewood, Mathematica (Cluj), 3 (1930), 33-48.

[14] J. Karamata: Sur un mode de croissance régulière des fonctions, Mathematica (Cluj), 4(1930), 38-53.

[15] J. Karamata: Sur un mode de croissance régulière, Théorèmes fondamentaux, Bull.Soc.Math. France, 61(1933), 55-62.

[16] J. Karamata: Bemerkung über die vorstehende Arbeit des Herrn Avakumović mit, näherer Betrachtung einer Klasse von Funktionen, welche bei den Inversionssätzen vorkommen, Bull. International, de l'Académie Yougoslave, Zagreb, 29 et 30 (1935), 117-123. (This is a contracted version of: A remark of the foregoing paper of $\mathrm{Mr} V$. Avakumović (in Serbian), Rad Jugoslovenske Akademije znanosti i umjetnosti, t. 254 (Razreda Matematičko-Prirodoslovnoga 79) (1936), 187- 200.)

[17] J. Karamata: Über die Hardy-Littlewoodsche Umkehrung des Abelschen Stätigkeitssatzes, Math. Zeitschrift, 32 (1930), 319-320.

[18] J. Karamata: Neuer Beweis und Verallgemeinerung der Tauberschen Sätze, welche die Laplacesche und Stieltjessche Transformation betreffen, Journal für die reine und angewandte Mathematik, 164 (1931), 27-39.

[19] J. Korevaar, T. van Aardenne - Ehrenfest, and N.G. de Bruijn: A note on slowly oscillating functions, Nieuw Arch. Wisk., 23 (1949), 77-86.

[20] J. E. Littlewood: The converse of Abel's theorem on power series, Proc. London Math. Soc. (2), 9 (1910), 434-448. 
[21] W. Matuszewska: Regularly increasing functions in connection with the theory of $L^{* \varphi}$-spaces, Studia Math., 21(1962), 317-344.

[22] W. Matuszewska: On a generalization of regularly increasing functions, Studia Math., 24 (1964), 271-279.

[23] W. Matuszewska, and W. Orlicz: On some classes of functions with regard to their orders of growth, Studia Math., 26(1965), 11-24.

[24] E. Omey: Multivariate reguliere variatie en toepassingen in kanstheorie, $\mathrm{Ph}$. D. Thesis, K. U. Leuven, 1982-Belgium. (Dutch).

[25] E. Omey, and E. Willekens: Second order behaviour of the tail of a subordinated probability distribution, Stoc. Proc. Appl., 21 (1986), 339-353.

[26] D. Djurčić: A statement of Tauber type for power series, Math. Moravica, 1 (1997), 33-34.

[27] R. Schmidt: Über divergente Folgen und lineare Mittelbildungen, Math. Zeitschrift, 22 (1925), 89-152.

[28] E. Seneta: Regularly Varying Functions, Lecture Notes in Mathematics 508, Springer - Verlag, Berlin - Heidelberg - New York, 1976.

[29] I. Schur: Zur Theorie der Cesaroschen und Hölderschen Mittelwerte, Math. Z., 31 (1930), 391-407.

[30] H. Steinhaus: Sur les distances des points de mesure positive, Fund. Math., 1 (1920), 93-104.

[31] J. L. Geluk, L. de Haan, and U. Stadtmüller: A Tauberian theorem of exponential type, Canadian J. Math., 38 (1986), 697-718.

[32] M. R. Tasković: Fundamental facts on translational $\mathcal{O}$-regularly varying functions, Math. Moravica, 7(2003), 107-152.

[33] M. R. Tasković: Survey on translational regularly varying functions, Mathematica (Cluj), 48 (71) (2006), 207-218.

[34] U. Stadtmüller, and R. Trautner: Tauberian theorems for Laplace transforms, Journal für die reine und angewandte mathematik, 310 (1979), 283-290.

[35] A. Tauber: Ein Satz aus der Theorie der unendlichen Reihen, Monatshefte für Mathematik und Physik, 8 (1897), 273-277.

Milan R. TASKović

FACULTY OF MATHEMATICS

11000 Belgrade, P.O. Box 550

SERBIA

Home Address:

Milan R. TASKOVić

Nehruova 236

11070 BELGRADE

SERBIA

E-mail address: andreja@predrag.us 